\begin{tabular}{|c|l|}
\hline Title & Optical Characterization of a Nanoscale Incommensurate Pitch in a New Liquid-Crystal Phase \\
\hline Author(s) & Cady, A.; Han, X.; OIson, D.; Orihara, Hiroshi; Huang, C. \\
\hline Citation & $\begin{array}{l}\text { Physical Review Letters, 91(12), 125502-1-125502-4 } \\
\text { https://doi.org/10.1103/PhysRevLett.91.125502 }\end{array}$ \\
\hline Issue Date & 2003-09 \\
\hline Doc URL & http://hdl.handle.net/2115/50768 \\
\hline Rights & ○ 2003 A merican Physical Society \\
\hline Type & article \\
\hline File Information & Phys. Rev. Lett. 91, 125502.pdf \\
\hline
\end{tabular}

Instructions for use 


\title{
Optical Characterization of a Nanoscale Incommensurate Pitch in a New Liquid-Crystal Phase
}

\author{
A. Cady ${ }^{1, *}$ X. F. Han, ${ }^{1}$ D. A. Olson, ${ }^{1}$ H. Orihara,${ }^{2}$ and C. C. Huang ${ }^{1}$ \\ ${ }^{1}$ School of Physics and Astronomy, University of Minnesota, Minneapolis, Minnesota 55455, USA \\ ${ }^{2}$ Department of Applied Physics, Nagoya University, Nagoya 464-8603, Japan
}

(Received 18 March 2003; published 16 September 2003)

\begin{abstract}
As a remarkable orientationally ordered soft-condensed matter system, the smectic- $C_{\alpha}^{*}$ phase exhibits an incommensurate nanoscale helical pitch of more than four smectic layers. Recent theoretical advances predict the existence of a new phase showing a helical pitch of less than four smectic layers $(<16 \mathrm{~nm})$. By applying a novel optical technique to the study of freestanding films with increments in thickness of one smectic layer, we have measured the size of this pitch using $633 \mathrm{~nm} \mathrm{HeNe} \mathrm{laser} \mathrm{light.}$ Our results confirm the existence of the newly predicted phase in one unique compound.
\end{abstract}

DOI: 10.1103/PhysRevLett.91.125502

PACS numbers: $61.30 . \mathrm{Gd}$

Liquid crystals provide scientists with many novel phases in which to study molecular interactions, structures, and physical properties in soft-condensed-matter systems. One liquid-crystal phase, the chiral smectic- $C_{\alpha}^{*}$ $\left(\mathrm{Sm}-C_{\alpha}^{*}\right)$, a Sm- $C^{*}$ variant phase [1], has been shown to exhibit an incommensurate nanoscale helical pitch (INHP). Like all Sm- $C^{*}$ variants, $\mathrm{Sm}-C_{\alpha}^{*}$ is a lamellar phase formed by rodlike molecules in which the molecular positions are liquidlike within the layers (1-layer $\approx$ $4 \mathrm{~nm})$. The molecules within each layer are oriented with their long axes along a common direction represented by a tilt $(\theta)$ relative to the layer normal and an azimuthal angle $(\psi)$. Aside from a $\mu \mathrm{m}$-scale modulation due to chiral interactions between neighboring layers, most $\mathrm{Sm}-C^{*}$ variant phases are found to exhibit a commensurate repeat unit in $\psi$ corresponding to an integer number of layers $(\leq 4)$. Chirality also allows polarizations to form within the layers, causing many phases to exhibit ferroelectric $(\mathrm{F})$ and antiferroelectric (AF) behaviors. In contrast, the INHP found in the $\mathrm{Sm}-C_{\alpha}^{*}$ phase has been measured to be as small as five molecular layers [2]. Recent theoretical advances [3] have predicted the existence of a new form of the $\mathrm{Sm}-C_{\alpha}^{*}$ phase exhibiting INHP structures of less than four layers, whereas only commensurate repeat units of four layers or less have been observed. To distinguish these two $\mathrm{Sm}-C_{\alpha}^{*}$ phases, we will use $\mathrm{Sm}-C_{\alpha 1}^{*}\left(\mathrm{Sm}-C_{\alpha 2}^{*}\right)$ for the phase with INHP $>4$ layers (INHP $<4$ layers). Typically, measuring such a small length scale in orientationally ordered systems is limited to resonant $\mathrm{x}$-ray scattering (RXS) [2] because the nanoscale structures are well beyond the diffraction limit in usual optical methods. By studying one unique compound, we establish the technique of using differential optical reflectivity (DOR) to determine the INHP. Moreover, our results lend strong support to the existence of the newly predicted $\mathrm{Sm}-C_{\alpha 2}^{*}$ phase.

The physical origin of order in chiral liquid crystals has been ascribed to frustration between interactions that favor incompatible ground states [4]. In the wellcharacterized Sm- $C_{\alpha 1}^{*}$ phase, the INHP results from frus- tration arising from competition between nearestneighbor (n.n.) F and next-nearest-neighbor (n.n.n.) AF interactions [5,6]. More specifically, the n.n. F interactions favor the Sm- $C^{*}$ structure, but the n.n.n. AF interactions favor a structure similar to the $\mathrm{Sm}-C_{\mathrm{F} / 2}^{*}$ phase [7]. In some compounds, the $\mathrm{F}$ interaction relieves this frustration on cooling through the Sm- $C_{\alpha 1}^{*}-\mathrm{Sm}-C^{*}$ transition in which a possibly continuous evolution of the helical pitch occurs from the $\mathrm{Sm}-C_{\alpha 1}^{*}$ (INHP $\sim 10$ layers) to the Sm- $C^{*}$ phase (pitch $\sim 100$ layers) $[6,8,9]$. In a different scenario, the n.n.n. AF interactions become greater with decreasing temperature, and the INHP decreases $[2,6]$. If the n.n. interaction becomes AF and the n.n.n. one remains AF of comparable strength, a phenomenological model predicts that the competition between these two interactions produces a new phase $\left(\mathrm{Sm}-C_{\alpha 2}^{*}\right)$ with the INHP being less than four layers [3].

Presumably, a proper starting point in the search for the $\mathrm{Sm}-C_{\alpha 2}^{*}$ phase would be to study a compound that exhibits a strong tendency toward n.n. AF ordering. Accordingly, we investigated the INHP structure in MHPOCBC, a compound exhibiting the phase sequence $\mathrm{Sm}-A\left(105.5^{\circ} \mathrm{C}\right) \mathrm{Sm}-C_{\alpha}^{*}\left(99.5^{\circ} \mathrm{C}\right) \mathrm{Sm}-C_{A}^{*}$. The chemical structure of MHPOCBC is shown at the top of Fig. 1. The presence of the direct Sm- $C_{\alpha}^{*}-\mathrm{Sm}-C_{A}^{*}$ phase transition suggests that the n.n. interaction may tend toward an AF nature in the $\mathrm{Sm}-C_{\alpha}^{*}$ phase, which is the criterion for the existence of the Sm- $C_{\alpha 2}^{*}$ phase.

Previous optical experiments on the $\mathrm{Sm}-C_{\alpha 1}^{*}$ phase supporting the INHP structure have included nulltransmission ellipsometry (NTE) [10-12], DOR [6,12], and observations of free surfaces on droplets [13]. Difficulties in studying the INHP arise from the fact that the $\mathrm{Sm}-C_{\alpha}^{*}$ phase is optically uniaxial in bulk, and so optical probes are insensitive to its structure. In freestanding films (FSFs), the surfaces are in general more ordered than the bulk and may exhibit a biaxial arrangement. The surfaces of FSFs in the Sm- $C_{\alpha 1}^{*}$ phase have been shown to have the same $\psi$ in adjacent layers (synclinic) $[6,11]$ or opposite $\psi$ in adjacent layers (anticlinic) $[6,10,12]$ 

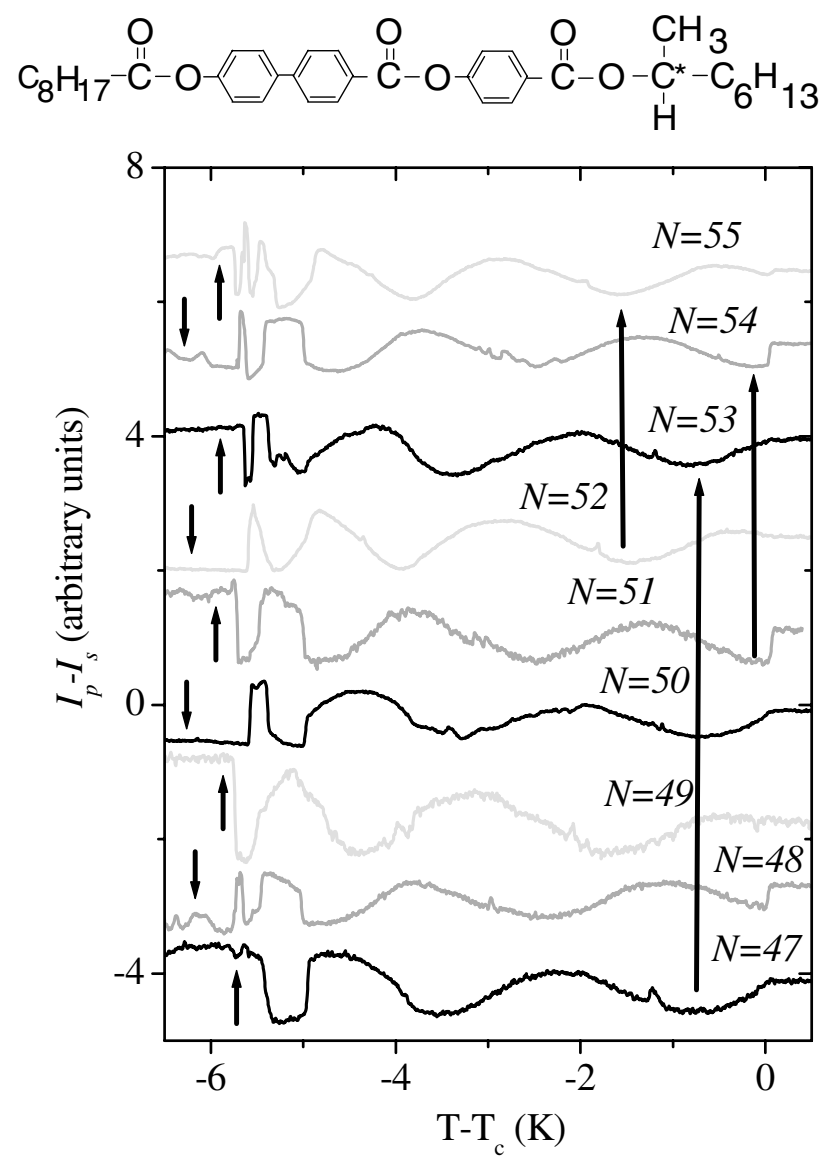

FIG. 1. The DOR signal versus $\left(T-T_{c}\right)$ for a series of films of MHPOCBC. Each temperature ramp was performed under the same direction of $E$ in the plane of the film. Long upward arrows illustrate periodicities of the signal with $N$ in the $\mathrm{Sm}-C_{\alpha 2}^{*}$ phase. The shorter arrows alternating in direction indicate the 2-layer periodicity of the data in the $\mathrm{Sm}-C_{A}^{*}$ phase. At the top is the chemical structure of MHPOCBC.

depending on the compound studied. Usually there exists a net polarization in the film due to the surface layers or an incomplete pitch, which allows the FSFs to be aligned by a small electric field. As the biaxial surfaces are linked to the INHP in the interior of the FSFs, DOR is sensitive to changes in the INHP through the biaxial surfaces. To determine the INHP and its temperature evolution using DOR, we require mainly (i) measurements of film thickness in number of layers $(N)$ and (ii) an optical probe that is sensitive to rotation of biaxial structures, i.e., the surfaces of FSFs.

Our thickness measurement scheme [14] has been developed to provide measurements of $N$ to within \pm 1 layer for films up to $N=100$ and \pm 2 layers for thicker films. To achieve this resolution in $N$, two lasers with wavelengths of 6328 and $5435 \AA$ are used to measure the reflectivity $(R)$ of the film at up to six difference incident angles in the Sm- $A$ phase. We simulate the resulting $R$ versus incidence angle curves using the $\mathrm{Sm}-A$ layer spacing $(d)$ and the ordinary index of refraction $\left(n_{o}\right)$, obtained from our NTE system [10]. For $N<100$ layers, we can easily differ- entiate incremental differences of one layer as shown clearly in Fig. 1.

As in other polarization-analyzed optical techniques, such as NTE, DOR measures the sample's effect on the polarization of light. In our DOR setup [6,14], incident $6328 \AA$ HeNe laser light is polarized by a GlanThompson polarizer mounted on a rotatable stage. After being reflected off the FSF, the light is divided by a polarizing beam splitter into $p$ and $s$ polarizations, where $p$ and $s$ denote polarizations parallel and perpendicular to the incidence plane, respectively. The acquired signals are the difference, $I_{p}-I_{s}$, and the sum, $I_{p}+I_{s}$, of the intensities. The experiment is begun with the film in the uniaxial Sm- $A$ phase. The Glan-Thompson polarizer is rotated to yield $I_{p}-I_{s}=0$. We have found that the $I_{p}-I_{s}$ signal is most sensitive to the optical properties of the FSF under these circumstances. Upon cooling and heating the film through the Sm- $C_{\alpha 2}^{*}$ phase we recorded $I_{p}-I_{s}$ and $I_{p}+I_{s}$ with typical ramps of $10-100 \mathrm{mK} / \mathrm{min}$. A small electric field $(E \approx 3 \mathrm{~V} / \mathrm{cm})$ was applied in the plane of the film to align the net polarization of the film.

In the INHP model of Sm- $C_{\alpha}^{*}$, the structure is optically uniaxial, but as described above, the surfaces layers can provide a net biaxiality in FSFs. In addition, partial windings of the INHP also add biaxiality to the FSF. In general, the INHP is by definition not an integer number of smectic layers, but as it evolves with temperature, the INHP will pass through values in which the ratio of the number of interior layers to the INHP is an integer. In this case the biaxiality of the interior of the FSF will be minimized. More important to changes in biaxiality of the FSF is the rotation of the surface layers linked to the INHP $[6,10,14]$ as the INHP evolves. The net result is that the biaxiality of the FSF will oscillate. Accordingly, the polarization of the reflected light will be altered, resulting in oscillations of the $I_{p}-I_{s}$ signal. The number of oscillations increases linearly with $N$, confirming that they are associated with changes to the interior of the FSF. Moreover, the linear variation of oscillations in $I_{p}-I_{s}$ with $N$ has a finite intercept [10], which allows us to determine approximately the total number of surface layers to be four.

To measure the INHP magnitude, DOR data are taken on a series of films with increments of one layer in thickness. The integer $N$ in a film along with the capability to determine $N$ allows us to use the smectic layer spacing as the benchmark for determining the INHP to within 0.5 layer. Data from MHPOCBC films with $N=47$ to $N=$ 55 are presented in Fig. $1 . T_{c}$ is the $\mathrm{Sm}-A-\mathrm{Sm}-C_{\alpha 2}^{*}$ transition temperature for MHPOCBC that was found from the $I_{p}+I_{s}$ signal where the onset of interior layer tilt is observed as a decrease in optical thickness of the film. The values of $I_{p}-I_{s}$ have been offset from each other for clarity.

One feature is that for some films, there is a substantial jump in $I_{p}-I_{s}$ at $T_{c}$, e.g., the $N=51$ film, while for 
others the change is much more subtle, e.g., the $N=52$ film. As described above, the DOR signal is mostly sensitive to the orientation of the surface layers. The two surfaces are in a planar arrangement in the $\mathrm{Sm}-A$ phase $[15,16]$, but may abruptly change to a nonplanar arrangement at $T_{c}$ as the INHP goes to a finite value at $T_{c}$. For some values of $N$ the initial INHP value leads to nearly planar surface layers, but in some cases the two surfaces are not planar, and a jump in $I_{p}-I_{s}$ occurs as the relative orientation of the surfaces changes at $T_{c}$ [17].

By identifying a periodicity in the slope or the jump in $I_{p}-I_{s}$, one can find the magnitude of the INHP directly below the Sm- $A-\mathrm{Sm}-C_{\alpha 2}^{*}$ transition. For example, the $N=51$ and $N=54$ films both show a similar jump in the $I_{p}-I_{s}$ signal at $T_{c}$, implying that the two surfaces are at the same relative orientation for both films. Accordingly, there has been one complete winding of the helix added between the $N=51$ and $N=54$ film, and thus the INHP is about three layers.

As the INHP changes with temperature, the relative orientation of the surface layers also changes. From the data the INHP can be determined because the orientation of the surfaces is related to whether $I_{p}-I_{s}$ is at an extrema or somewhere in between. For instance, every time the signal is at a maximum, the two surfaces have the same relative orientation. By similar reasoning, the INHP can be determined at any temperature by identifying a periodicity in the slope of the $I_{p}-I_{s}$ signal as a function of $N$. Since $N$ is quantized, the INHP is easily known to within $\frac{1}{2}$ layer. In Fig. 1 , arrows are drawn between DOR data with equivalent slopes from the $N=$ 51 and $N=54$ films and the $N=52$ and $N=55$ films, indicating an INHP of $\approx 3$ layers. Moreover, the arrow joining the $N=47$ and $N=53$ films indicates that the INHP is closer to 3 layers than to 2.5 or 3.5 layers. If the INHP were closer to 2.5 layers, the periodicity would appear between films differing in $N$ by 5 layers.

At least two additional pieces of information can be gathered from the DOR data. First, the two-layer periodicity of the $\mathrm{Sm}-C_{A}^{*}$ phase can be seen below the Sm- $C_{\alpha 2}^{*}$ phase in Fig. 1. In films of even $N$ (e.g., $N=$ 50 ), there is a step down in the DOR signal at the $\mathrm{Sm}-C_{\alpha 2}^{*}-\mathrm{Sm}-C_{A}^{*}$ transition whereas in films of odd $N$, e.g., $N=53$, there is a step up in the signal. The data close to the transition are noisy and not reproducible, indicating a possible two-phase coexistence. Second, upon reversing the direction of $E$, the DOR signal remains largely unchanged, indicating an AF response, associated with an anticlinic surface structure. For brevity, only data from one orientation of $E$ are shown in Figs. 1 and 2.

The $4 \times 4$ matrix method was used to simulate our data [18]. The solid lines in Fig. 2 are the simulation results. Three parameters, $d, n_{o}$, and the extraordinary index of refraction $n_{e}$ used in the simulations were obtained from our NTE. For MHPOCBC $d=36.0 \pm 0.1 \AA$, $n_{o}=1.477 \pm 0.005$, and $n_{e}=1.633 \pm 0.010$. All the

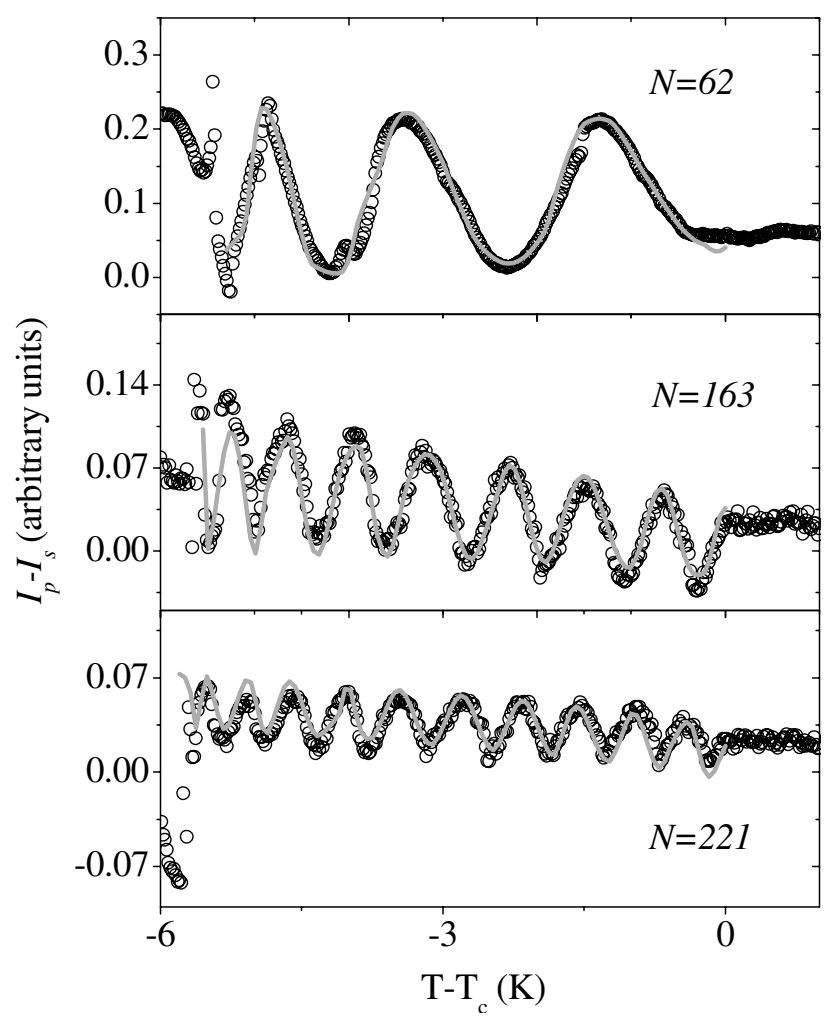

FIG. 2. DOR data (circles) acquired from $N=62, N=163$, and $N=221$ MHPOCBC films while cooling at $80 \mathrm{mK} / \mathrm{min}$ with $E=3 \mathrm{~V} / \mathrm{cm}$ in the plane of the film. Simulated results are shown as solid gray lines.

MHPOCBC data are fitted well using two anticlinic layers on each surface.

The temperature variation of the INHP was found by simulating the data until the simulated $I_{p}-I_{s}$ values yielded the best match to the measured ones. In particular, the positions of the extrema in the $I_{p}-I_{s}$ strongly depend on the temperature variation of pitch. The amplitude of the oscillations is determined by the film reflectivity and the biaxiality of the structure associated with the interior and surface tilt. The relatively small continuous change in this amplitude for a given film demonstrates that the number of surface layers is constant, thus the fits were done without varying the number of surface layers. The tilt can be found by fitting $I_{p}+I_{s}$ at the experimental angle of incidence. The drawing in Fig. 3 depicts the INHP structure with anticlinic surfaces used to model the data. The gray ellipsoids represent an average molecule in each of the layers, which are represented by projections of circles in the drawing.

The INHP temperature evolution for MHPOCBC is plotted in Fig. 3 for the $N=62,163$, and 221 films. The INHP decreases on cooling from $\approx 3$ to $\approx 2.6$ layers. Although the error on the starting value of INHP is $<0.5$ layers, the relative resolution of the INHP is well within 0.1 layers. The fitting to the DOR data as shown in Fig. 2 is strongly dependent on the INHP used. Furthermore, the INHP temperature evolutions used to 




FIG. 3. The plot shows the pitch evolutions used in simulating data from the $N=62$ (light gray line), $N=163$ (dark gray line), and $N=221$ (black line) films. Stars represent NTE measurements of the pitch in the Sm- $C_{A}^{*}$ phase. On the right is a cartoon of the INHP structure with ellipsoids representing molecules. $N_{s} \approx 2$ is the number of anticlinic surface layers.

fit films of various thickness were roughly equivalent, and the data are representative of the over 12 films studied. Modeling the DOR data from the opposite direction of $E$ yields the same INHP evolution. In addition, the pitch of the $\mathrm{Sm}-C_{A}^{*}$ phase obtained from our NTE is shown as stars in Fig. 3. This pitch is the combination of a basic 2-layer structure and an optical pitch with a length of $\approx 50$ layers.

By studying a liquid-crystal compound with a direct $\mathrm{Sm}-C_{\alpha 2}^{*}-\mathrm{Sm}-C_{A}^{*}$ transition, we have confirmed the existence of an INHP with a length of less than four layers as predicted by a recent theoretical model [3]. The INHP decreases in magnitude from $\approx 3$ layers on cooling through the Sm- $C_{\alpha 2}^{*}$ phase. As discussed at the beginning of the paper, some systems with a direct $\mathrm{Sm}-C_{\alpha 1}^{*}-\mathrm{Sm}-C^{*}$ transition exhibit an INHP that evolves continuously into the longer pitch observed in the $\mathrm{Sm}-C^{*}$. In that case the frustration was relieved by the n.n.n. AF interaction becoming small compared to the n.n. F interaction. In the case of the direct Sm- $C_{\alpha 2}^{*}-\mathrm{Sm}-C_{A}^{*}$, the INHP decreases but does not continuously evolve into the 2-layer pitch in the $\mathrm{Sm}-C_{A}^{*}$ phase. The irreproducibility of the data near this transition suggests a coexistence region and a firstorder transition. One important question to be addressed experimentally is the following: are the $\mathrm{Sm}-C_{\alpha 1}^{*}$ phase found in compounds with INHP $>4$ layers and the $\mathrm{Sm}-C_{\alpha 2}^{*}$ with INHP $<4$ layers miscible; that is, are they the same type of phase? Such experimental work requires larger quantities of samples for studying various mixtures. In the phase diagram generated by the n.n. versus n.n.n. interaction strengths, these two phases are separated. The $\mathrm{Sm}-\mathrm{C}_{\alpha 2}^{*}$ phase is the result of competing n.n. AF and n.n.n. AF interactions. The INHP structure relieves the frustration. As the n.n. interaction strength becomes sufficiently weak, the theoretical model yields a stable 4-layer structure, e.g., the $\mathrm{Sm}-C_{F I 2}^{*}$ phase, which has approximately n.n.n. AF coupling. The phase with a 4-layer structure separates two $\mathrm{Sm}-C_{\alpha}^{*}$ phases with pitch $>4$ layers and pitch $<4$ layers.

In conclusion, we have developed and refined a technique that allows the measurement of molecular orientational periodicities as small as $\approx 10 \mathrm{~nm}$ using visible laser light. The application of this technique to a chiral liquid-crystal compound has revealed an INHP of an unusually short length. This newly measured INHP adds further support to a theoretical model for describing molecular ordering in the chiral $\mathrm{Sm}-\mathrm{C}^{*}$ variant phases [3].

We thank Showa Shell Sekiyu Co., Ltd. for supplying the MHPOCBC. A. C. wishes to acknowledge financial support from the Stanwood Johnston Memorial Foundation. This research was supported in part by the National Science Foundation, Solid State Chemistry Program under Grants No. DMR-0106122 and No. 9901739.

*Present address: Advanced Photon Source, Argonne National Laboratory, Argonne, Illinois 60439.

[1] M. Fukui, H. Orihara, Y. Yamada, N. Yamamoto, and Y. Ishibashi, Jpn. J. Appl. Phys. 28, L849 (1989).

[2] P. Mach et al., Phys. Rev. E 60, 6793 (1999).

[3] D. A. Olson, X. F. Han, A. Cady, and C. C. Huang, Phys. Rev. E 66, 021702 (2002).

[4] R. Kamien and J. V. Selinger, J. Phys. Condens. Matter 13, R1 (2001).

[5] M. Čepič and B. Žekš, Mol. Cryst. Liq. Cryst. 263, 61 (1995).

[6] A. Cady, D. A. Olson, X. F. Han, H. T. Nguyen, and C. C. Huang, Phys. Rev. E 65, 030701 (2002).

[7] A. Cady et al., Phys. Rev. E 64, 050702(R) (2001).

[8] L. S. Hirst et al., Phys. Rev. E 65, 041705 (2002).

[9] C. Da Cruz, J. C. Rouillon, J. P. Marcerou, N. Isaert, and H. T. Nguyen, Liq. Cryst. 28, 125 (2001).

[10] P. M. Johnson, S. Pankratz, P. Mach, H. T. Nguyen, and C. C. Huang, Phys. Rev. Lett. 83, 4073 (1999).

[11] D. Schlauf, Ch. Bahr, and H. T. Nguyen, Phys. Rev. E 60, 6816 (1999).

[12] D. A. Olson, S. Pankratz, P. M. Johnson, A. Cady, H. T. Nguyen, and C. C. Huang, Phys. Rev. E 63, 061711 (2001).

[13] V. Laux, N. Isaert, G. Joly, and H. T. Nguyen, Liq. Cryst. 26, 361 (1999).

[14] S. Pankratz, P. M. Johnson, and C. C. Huang, Rev. Sci. Instrum. 71, 3184 (2000).

[15] D. Schlauf, Ch. Bahr, V. K. Dolganov, and J.W. Goodby, Eur. Phys. J. B 9, 461 (1999).

[16] D. R. Link, G. Natale, N. A. Clark, J. E. Maclennan, M. Walsh, S.S. Keast, and M. E. Neubert, Phys. Rev. Lett. 82, 2508 (1999).

[17] The $I_{p}+I_{s}$ data show no jump at the Sm- $A-\mathrm{Sm}-C_{\alpha 2}^{*}$ transition. This agrees with previous heat capacity results that yield a continuous $\mathrm{Sm}-A-\mathrm{Sm}-C_{\alpha 2}^{*}$ transition in MHPOCBC. See R. Ema and H. Yao, Phys. Rev. E 57, 6677 (1998).

[18] D.W. Berreman, J. Opt. Soc. Am. 62, 502 (1972). 